\title{
Modelica Component Models for Oceanic Surface Waves and Depth Varying Current
}

\author{
Savin Viswanathan ${ }^{1}$ Christian Holden ${ }^{1}$ \\ ${ }^{1}$ Dept. of Mechanical and Industrial Engineering, Norwegian University of Science and Technology (NTNU), \\ NO-7491 Trondheim, Norway. \{savin.viswanathan, christian.holden\}@ntnu.no
}

\begin{abstract}
In this paper, the theory of progressive ocean-surface gravity-waves is discussed, followed by the concept of the representation of the irregular sea-state by a sea-spectrum. Fourier series decomposition of the irregular sea-surface into its constituent regular waves and the method of realizing unique time-records of the sea-surface-elevation from commonly used sea-spectra is described. A detailed description of the development of Modelica componentmodels to generate regular as well as irregular waves, and depth-varying current, with an eye on the requirements imposed by probable integrated simulation scenarios, is then presented and the results discussed.
\end{abstract}

Keywords: regular wave, irregular wave, sea-spectrum, Modelica ocean-engineering library.

\section{Introduction}

The advantages of developing an OpenModelica ocean engineering library populated with domain-specific component-models and functions to carry out the integrated simulation of multi-pyhsical ocean engineering systems was demonstrated by the authors (Viswanathan and Holden, 2019). This earlier work:

1. Gives a brief description of the simulation of systems based on the hydrodynamic response of catenarymoored non-diffracting floating objects in the presence of waves and current,

2. Demonstrates the satisfactory agreement of the Modelica simulation results with those obtained using a popular ocean-engineering commercial software (Orcaflex), and

3. Brings out the advantages of using a componentmodel based simulation approach.

The voluminous nature of the earlier work precluded the possibility of delving into the theoretical and implementational details of the various Modelica componentmodels of the ocean-engineering library proposed by the authors, the preliminary version of which is available for download at github.com/Savin-Viswanathan/ OELib_OMAE2019.
The present work which deals with the development of Modelica component-models for simulating the kinematics and dynamics of regular and irregular waves, and depth varying current, is the first among a series of two papers which will fill in such gaps in theory and implementation.

\section{Theory}

The theory presented here upto Section 2.4.2 is a brief summary of that given in (Dean and Dalrymple, 2001) .

\subsection{The Fundamentals}

The application of the conservation of mass to a reference fluid volume yields the continuity equation:

$$
\begin{aligned}
\frac{1}{\rho}\left(\frac{\partial \rho}{\partial t}+\right. & \left.u \frac{\partial \rho}{\partial x}+v \frac{\partial \rho}{\partial y}+w \frac{\partial \rho}{\partial z}\right) \\
& +\frac{\partial u}{\partial x}+\frac{\partial v}{\partial y}+\frac{\partial w}{\partial z}=0 .
\end{aligned}
$$

Here, $\rho\left[\mathrm{kg} / \mathrm{m}^{3}\right]$ is the fluid density, $t[\mathrm{~s}]$ is time, and $u, v, w$ $[\mathrm{m} / \mathrm{s}]$ are the fluid velocities in the $x, y, z$ directions.

Disregarding the effects of surface tension and elasticity, the application of the translational equation of motion to a fluid particle yields the Navier-Stoke's equations:

$$
\begin{aligned}
\frac{\mathrm{D} u}{\mathrm{D} t} & =-\frac{1}{\rho} \frac{\partial p}{\partial x}+\frac{1}{\rho}\left(\frac{\partial \tau_{x x}}{\partial x}+\frac{\partial \tau_{y x}}{\partial y}+\frac{\partial \tau_{z x}}{\partial z}\right)+X \\
\frac{\mathrm{D} v}{\mathrm{D} t} & =-\frac{1}{\rho} \frac{\partial p}{\partial y}+\frac{1}{\rho}\left(\frac{\partial \tau_{x y}}{\partial x}+\frac{\partial \tau_{y y}}{\partial y}+\frac{\partial \tau_{z y}}{\partial z}\right)+Y \\
\frac{\mathrm{D} w}{\mathrm{D} t} & =-\frac{1}{\rho} \frac{\partial p}{\partial z}+\frac{1}{\rho}\left(\frac{\partial \tau_{x z}}{\partial x}+\frac{\partial \tau_{y z}}{\partial y}+\frac{\partial \tau_{z z}}{\partial z}\right)+Z
\end{aligned}
$$

Here, $\frac{\mathrm{D}}{\mathrm{D} t}$ is the material derivative, $p\left[\mathrm{~N} / \mathrm{m}^{2}\right]$ is the fluid pressure, $\tau\left[\mathrm{N} / \mathrm{m}^{2}\right]$ is the shear stress where the first subscript refers to the surface perpendicular and the second subscript refers to the direction of the stress, and $X, Y, Z$ $[\mathrm{N}]$ are body forces along the $x, y$, and $z$ directions.

\subsection{Assumptions and the Governing Equation}

The following assumptions are made:

- Incompressible fluid ( $\rho=$ constant).

- Inviscid fluid $(\tau=0)$.

- Irrotational flow $\left(\frac{\partial w}{\partial y}=\frac{\partial v}{\partial z}, \frac{\partial w}{\partial x}=\frac{\partial u}{\partial z}\right.$ and $\left.\frac{\partial v}{\partial x}=\frac{\partial u}{\partial y}\right)$. 
- Low wave steepness, i.e., $(H \ll L)$.

- Long crested waves (2D flow, in $x$ and $z$ directions only).

- Horizontal and time-invariant bottom boundary.

Assuming incompressible fluid and long crested waves,

$$
\text { Eqn. (1) } \rightarrow \frac{\partial u}{\partial x}+\frac{\partial w}{\partial z}=0
$$

Assumption of inviscid fluid gives the Euler equations:

$$
\begin{aligned}
& \text { Eqn. (2) } \rightarrow \frac{\mathrm{D} u}{\mathrm{D} t}=-\frac{1}{\rho} \frac{\partial p}{\partial x}, \\
& \text { Eqn. (4) } \rightarrow \frac{\mathrm{D} w}{\mathrm{D} t}=-\frac{1}{\rho} \frac{\partial p}{\partial z}-g .
\end{aligned}
$$

The assumption of irrotational flow makes it possible to define a scalar velocity potential $\phi(x, y, z, t)\left[\mathrm{m}^{2} / \mathrm{s}\right] \mathrm{such}$ that its directional derivative gives the fluid velocity in that direction. i.e.,

$$
u=\frac{\partial \phi}{\partial x}, \quad w=\frac{\partial \phi}{\partial z} .
$$

Thus, for an incompressible, irrotational flow in the $x$ and $z$ directions, the integrated form of the Euler equation yields the Bernoulli equation for unsteady potential flow,

$$
\frac{\partial \phi}{\partial t}+\frac{1}{2}\left\{\left(\frac{\partial \phi}{\partial x}\right)^{2}+\left(\frac{\partial \phi}{\partial z}\right)^{2}\right\}+\frac{p}{\rho}+g z=C(t)
$$

where $C(t)$ is the Bernoulli term and is a constant for steady flows.

With $\mathbf{u}=[u, w]^{\mathrm{T}},(5)$ may be expressed in vector form as $\nabla . \mathbf{u}=0$. From (8), $\mathbf{u}=\nabla \phi$, so we have

$$
\nabla . \nabla \phi=\nabla^{2} \phi=\frac{\partial^{2} \phi}{\partial x^{2}}+\frac{\partial^{2} \phi}{\partial z^{2}}=0
$$

Equation (10) is the well-known Laplace equation, and consitutes the governing differential equation which is valid throughout the fluid domain. Our interest is in determining the velocity potential which satisifies the Laplace equation, which then makes it possible to determine the fluid velocities at any point in the fluid domain.

The Bernoulli equation relates the fluid velocities to the the fluid pressure, and the integration of the fluid pressure along the surface of any submerged/floating object gives the force that the fluid exerts on the object, which is, in most cases, the element of interest in wave-body interaction problems.

\subsection{The Boundary Conditions}

In seeking a solution for the velocity potential in (10), we make use of the following physical conditions which must be satisfied by the fluid velocity and pressure, at the domain boundaries:

1. The Kinematic Free-Surface Boundary-Condition (KFSBC) stemming from the fact that there cannot be any fluid flow across the interface between the liquid domain and the atmosphere at the free surface of the fluid.

2. The Bottom Boundary-Condition (BBC) stemming from the fact that there cannot be any fluid flow across the sea floor.

3. The Dynamic Free-Surface Boundary-Condition (DFSBC) stemming from the fact that 'free' surfaces such as the air-water interface cannot support pressure variations across it, and hence must be capable of responding in order to maintain the pressure continuity across the liquid and gaseous domains. This displacement of the free surface means that the position of the upper boundary is not known a priori in the water-wave problem. For small-amplitude waves, this condition is given by the requirement that the pressure on the free surface is uniform along the wave form.

4. The Spatial and Temporal Periodicity Condition at the Lateral Surfaces (LPBC) stemming from the fact that the solution we seek is the velocity potential associated with a wave which is periodic in both space and time.

Mathematical expressions for the kinematic boundary conditions may be derived from the equation of the form $\mathrm{F}(x, y, z, t)=0$, describing the boundary surface. For a temporally varying surface, the total time-derivative of the surface is zero, on the surface. Hence, for a $2 \mathrm{D}$ wave surface-profile,

$$
\frac{\mathrm{DF}}{\mathrm{D} t}=\frac{\partial \mathrm{F}}{\partial t}+u \frac{\partial \mathrm{F}}{\partial x}+w \frac{\partial \mathrm{F}}{\partial z}=0 \text { on } \mathrm{F}(x, z, t)=0 .
$$

Or, rearranging and using vector notation,

$$
-\frac{\partial \mathrm{F}}{\partial t}=\mathbf{u} \cdot \nabla \mathrm{F}=\mathbf{u} \cdot \mathbf{n}|\nabla \mathrm{F}|
$$

Here, $\mathbf{n}=\frac{\nabla \mathrm{F}}{|\nabla \mathrm{F}|}$ is the unit normal to the surface, and $|\nabla \mathrm{F}|=\sqrt{\left(\frac{\partial \mathrm{F}}{\partial x}\right)^{2}+\left(\frac{\partial \mathrm{F}}{\partial z}\right)^{2}}$

The kinematic boundary condition is thus expressed as

$$
\mathbf{u . n}=-\frac{\partial \mathrm{F} / \partial t}{|\nabla \mathrm{F}|} \text { on } \mathrm{F}(x, y, z, t)=0 .
$$


At the free surface, $\mathrm{F}(x, z, t)=z-\eta(x, t)=0$, where $\eta(x, t)$ is the displacement of the free surface about the horizontal plane. Equation (13) gives

$$
\mathbf{u . n}=\frac{\partial \eta / \partial t}{\sqrt{\left(\frac{\partial \eta}{\partial x}\right)^{2}+1}} \text { on } z-\eta(x, t)=0 .
$$

Taking

$$
\mathbf{n}=\frac{-\frac{\partial \eta}{\partial x} \mathbf{i}+\mathbf{k}}{\sqrt{\left(\frac{\partial \eta}{\partial x}\right)^{2}+1}},
$$

(14) gives the KFSBC as

$$
w=\frac{\partial \eta}{\partial t}+u \frac{\partial \eta}{\partial x} \text { on } z=\eta(x, t) .
$$

Assuming a horizontal, time-invariant bottom at $z=$ $-h, \mathrm{~F}(z)=z+h=0$. Equation (13) gives u.n $=0$ at $z=-h$. Here, $\mathbf{n}=\mathbf{k}$, and hence the BBC can be expressed as

$$
w=0 \text { on } z=-h \text {. }
$$

By specifying a uniform pressure $\left(p_{\eta}=\right.$ constant $)$ along the wave form in the Bernoulli equation at the free surface, the DFSBC may be expressed mathematically as

$$
\begin{aligned}
\frac{\partial \phi}{\partial t}+\frac{1}{2}\left\{\left(\frac{\partial \phi}{\partial x}\right)^{2}+\right. & \left.\left(\frac{\partial \phi}{\partial z}\right)^{2}\right\} \\
& +\frac{p_{\eta}}{\rho}+g z=\mathrm{C}(t) \text { on } z=\eta(x, t) .
\end{aligned}
$$

The LPBCs may be expressed as

$$
\begin{aligned}
& \phi(x, t)=\phi(x+L, t), \\
& \phi(x, t)=\phi(x, t+T) .
\end{aligned}
$$

Here, $L[\mathrm{~m}]$ is the wave length and $T[\mathrm{~s}]$ is the wave period.

\subsection{Solution of the Boundary Value Problem}

The BVP to be solved is thus the Laplace equation (10) subject to: 1. the KFSBC in (16), 2. the DFSBC in (18), 3 . the BBC in (17), and the LPBC in (19) and (20). The diagrammatic representation of the problem is shown in Figure 1.

\subsubsection{Manipulation of the Free Surface Boundary Conditions}

On carrying out a non-dimensional analysis of the terms in the KFSBC and the DFSBC, under the assumption of low wave steepness, i.e., $H / L \ll 1$, we notice that $u \frac{\partial \eta}{\partial x} \ll \frac{\partial \eta}{\partial t}$, $u \frac{\partial \eta}{\partial x} \ll \frac{\partial \phi}{\partial z}$ and $\left(\frac{\partial \phi}{\partial x}\right)^{2},\left(\frac{\partial \phi}{\partial z}\right)^{2} \ll \frac{\partial \phi}{\partial t}$.

Further, the KFSBC and DFSBC are to be evaluated at $z=\eta(x, t)$, which is a priori unknown. However, on taking

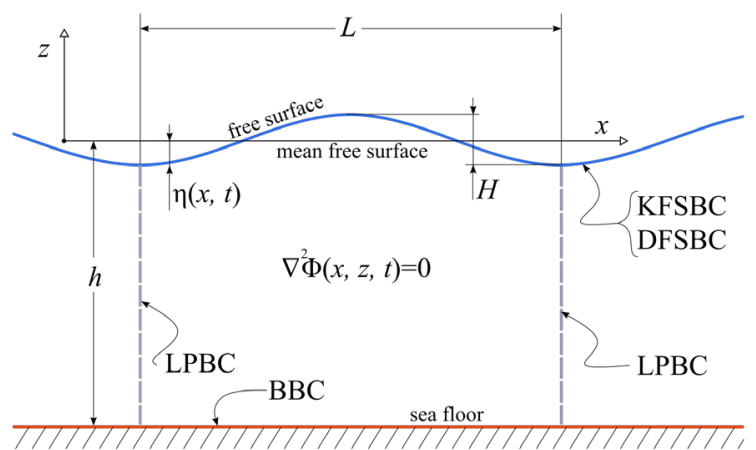

Figure 1. Boundary value problem for the velocity potential. Adapted from (Dean and Dalrymple, 2001).

a Taylor series expansion of the BCs about the mean free surface at $z=0$, we notice that the second-order and subsequent higher-order terms can be neglected; hence, we can safely assume the validity of the BCs at the mean free surface instead of the actual free surface. Details about linearization and shifting can be found in (Techet, 2005).

Taking the pressure at the free surface as the constant atmospheric pressure, we can eliminate the Bernoulli constant and the pressure term in (9) as demonstrated in (Andersen and Frigaard, 2011). Thus, the modified BCs are:

$$
\begin{array}{ll}
\text { KFSBC: } & \frac{\partial \phi}{\partial z}=\frac{\partial \eta}{\partial t} \quad \text { on } \quad z=0, \\
\text { DFSBC: } & \frac{\partial \phi}{\partial t}+g \eta=0 \quad \text { on } \quad z=0 .
\end{array}
$$

Differentiating (22) w.r.t. $t$ and using (21), we can combine both the BCs to give the Combined Free-Surface Boundary-Condition (CFSBC) as:

$$
\text { CFSBC: } \quad \frac{\partial^{2} \phi}{\partial t^{2}}+g \frac{\partial \phi}{\partial z}=0 \quad \text { on } \quad z=0 \text {. }
$$

\subsubsection{Complex Exponential Form of the Velocity Po- tential}

It is often mathematically advantageous to use the complex form of the velocity potential; see p. 4 of (Chakrabarti, 1987). Since the solution we seek is related to a progressive sinusoidal wave, we may express the velocity potential as

$$
\phi=\varphi(z) e^{i(k x-\omega t)}
$$

The LPBCs were utilized in the formulation of the above equation, the real part of which represents the velocity potential of a sinusoidal wave progressing in the positive $\mathrm{x}$ direction; see pp. 2, 12 of (Krogstad and Arntsen, 2000). 
The BVP is now given by:

$$
\begin{array}{ll}
\text { Laplace eqn: } & \frac{\partial^{2} \varphi}{\partial z^{2}}-k^{2} \varphi=0 . \\
\text { CFSBC: } & -\omega^{2} \varphi+g \frac{\partial \varphi}{\partial z}=0 \text { on } z=0 . \\
\text { BBC: } & \frac{\partial \varphi}{\partial z}=0 \text { on } z=-h .
\end{array}
$$

Assuming a solution of the form

$$
\begin{aligned}
& \qquad \varphi(z)=\mathrm{C}_{1} e^{-k z}+\mathrm{C}_{2} e^{k z}, \\
& \text { Eqn. (27) } \rightarrow \quad \mathrm{C}_{1} k e^{-k h}-\mathrm{C}_{2} k e^{k h}=0, \\
& \text { Eqn. (26) } \rightarrow \quad\left(g k-\omega^{2}\right) \mathrm{C}_{1}-\left(g k+\omega^{2}\right) \mathrm{C}_{2}=0 .
\end{aligned}
$$

The above homogenous equation system has non-trivial solutions only when the determinant is zero. This gives the dispersion relation

$$
\omega^{2}=g k \tanh (k h)
$$

Now, setting $\mathrm{C}_{1}=\frac{1}{2} \mathrm{~B} e^{k h}$ and $\mathrm{C}_{2}=\frac{1}{2} \mathrm{~B} e^{-k h}$,

$$
\begin{aligned}
& \text { Eqn. }(28) \rightarrow \quad \varphi(z)=B \cosh [k(z+h)], \\
& \text { Eqn. }(24) \rightarrow \quad \phi(x, z, t)=B \cosh [k(z+h)] e^{i(k x-\omega t)}
\end{aligned}
$$$$
\text { Eqn. }(22) \rightarrow \quad \eta=\left.\frac{-1}{g} \frac{\partial \phi}{\partial t}\right|_{z=0}
$$

Considering that that highest value of $\eta$ is the wave amplitude $A=H / 2$ [m], from (33) and (34), we have

$$
B=\frac{-i g A}{\omega} \frac{1}{\cosh (k h)}
$$

The complex exponential form of the velocity potential may now be expressed as

$$
\phi(x, z, t)=\frac{-i g A}{\omega} \frac{\cosh k(z+h)}{\cosh (k h)} e^{i(k x-\omega t)}
$$

\subsection{Kinematics and Dynamics of Regular Waves}

Considering the real part of the velocity potential in (36),

$$
\begin{aligned}
\phi & =\frac{g H}{2 \omega} \frac{\cosh k(z+h)}{\cosh (k h)} \sin (k x-\omega t), \\
\eta & =H / 2 \cos (k x-\omega t), \\
u & =\frac{\pi H}{T} \frac{\cosh k(z+h)}{\sinh (k h)} \cos (k x-\omega t), \\
w & =\frac{\pi H}{T} \frac{\sinh k(z+h)}{\sinh (k h)} \sin (k x-\omega t), \\
\dot{u} & =\frac{2 \pi^{2} H}{T^{2}} \frac{\cosh k(z+h)}{\sinh (k h)} \sin (k x-\omega t), \\
\dot{w} & =-\frac{2 \pi^{2} H}{T^{2}} \frac{\sinh k(z+h)}{\sinh (k h)} \cos (k x-\omega t), \\
\delta_{x} & =-\frac{H}{2} \frac{\cosh k(z+h)}{\sinh (k h)} \sin (k x-\omega t), \\
\delta_{z} & =\frac{H}{2} \frac{\sinh k(z+h)}{\sinh (k h)} \cos (k x-\omega t), \\
p & =\rho g \frac{H}{2} \frac{\cosh k(z+h)}{\cosh (k h)} \cos (k x-\omega t) .
\end{aligned}
$$

Here, $\dot{u}$ and $\dot{w}\left[\mathrm{~m} / \mathrm{s}^{2}\right]$ are the water particle accelerations, $\delta_{x}$ and $\delta_{z}[\mathrm{~m}]$ are water particle displacements from their mean position, and $p\left[\mathrm{~N} / \mathrm{m}^{2}\right]$ is the dynamic pressure. (Chakrabarti, 1987).

\subsection{Kinematics and Dynamics of Irregular Waves}

The process of linearization carried out in Section 2.4.1 implies the validity of the superpostion principle with regards to the quantities expressed in Section 2.5. This, in turn, justifies the representation of the irregular wave parameters as the summation of the parameters of constituent regular waves; or, in other words, as a Fourier series, as represented in Figure 2.

The way of describing the sea-state is linked to the energy content in waves. Linear theory gives the wave energy per unit area of the sea surface due to a regular wave as

$$
E=\frac{1}{2} \rho g \zeta_{0 i}^{2}
$$

Here, $\zeta_{0 i}[\mathrm{~m}]$ is the amplitude of the regular wave under consideration; see p. 97 of (Dean and Dalrymple, 2001).

The spectrum for the irregular wave process is defined such that the area of the wave spectrum $S_{\eta}(\omega)$ within the frequency interval $\Delta \omega$ represents the wave energy for the same frequency interval. Hence, from a known spectrum function, we can find the amplitude $\zeta_{0 i}[\mathrm{~m}]$ of the harmonic wave component which represents the wave energy for a given frequency resolution using (47); see p. 23 of (Faltinsen, 1999), and p. 122 of (Chakrabarti, 1987):

$$
\zeta_{0 i}=\sqrt{2 S_{\eta}\left(\omega_{i}\right) \Delta \omega}
$$




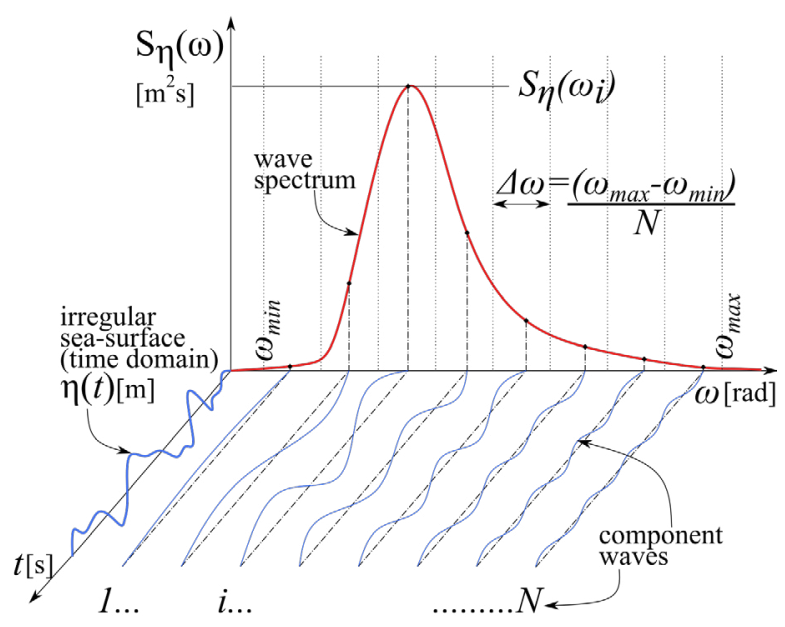

Figure 2. Concept of Fourier series representation of irregular waves and the wave spectrum. Adapted from (Faltinsen, 1999).

Once the amplitudes corresponding to each of the component regular waves is known, randomness may be introduced by the inclusion of an arbitrary phase difference $\varepsilon_{i}[\mathrm{rad}]$. The property of the irregular wave may now be expressed as the summation of the property of the component waves of specified frequencies with random phase. For e.g., the sea surface elevation (SSE) at a given $x$ coordinate is expressed by equation (48); see p. 123 of (Chakrabarti, 1987):

$$
\eta(x, t)=\sum_{i=1}^{N} \zeta_{0 i} \cos \left(k_{i} x-\omega_{i} t-\varepsilon_{i}\right) .
$$

Here, $N$ is the total number of wave components (frequency bands), $\zeta_{0}[\mathrm{~m}]$ is the component wave amplitude, $\omega[\mathrm{rad} / \mathrm{s}]$ is the wave angular frequency, and $\varepsilon[\mathrm{rad}]$ is the phase. Subscript $i$ refers to the number of the component wave under consideration. The wave number $k[\mathrm{rad} / \mathrm{m}]$ is to be determined from the dispersion relation given in (31). $g\left[\mathrm{~m} / \mathrm{s}^{2}\right]$ is the acceleration of gravity and $d[\mathrm{~m}]$ is the water depth. $S_{\eta}\left(\omega_{i}\right)\left[\mathrm{m}^{2} \mathrm{~s}\right]$ is the energy spectral density and $\Delta \omega[\mathrm{rad} / \mathrm{s}]$ is the width of the frequency bands dividing the total wave spectrum.

The even distribution of component frequencies will cause the resultant wave to be periodic with a period of of $2 \pi / \omega_{\min }$ [s], and thus not truly irregular. Hence, the component frequency within each frequency interval is selected based on a unifrom random distribution, as advised in p. 209 of (Fossen, 2011).

\section{Modelica Implementation}

\subsection{General Considerations}

Most simulation problems envisaged would require, in one way or the other, the determination of wave/current forces acting on structures with varying degrees of restraint as illustrated in Figure 3. Since the wave forces vary both temporally and spatially, and since the location information is contained in the component-model for the body in a wave-body interaction problem, it was decided that a wave component-model that generates all the required parameters that allows for the determination of the various quantities given in the equations under Section 2.5, at any location $(x, y, z)$ within the problem domain, at any specified simulation time $t$, would be the best approach.

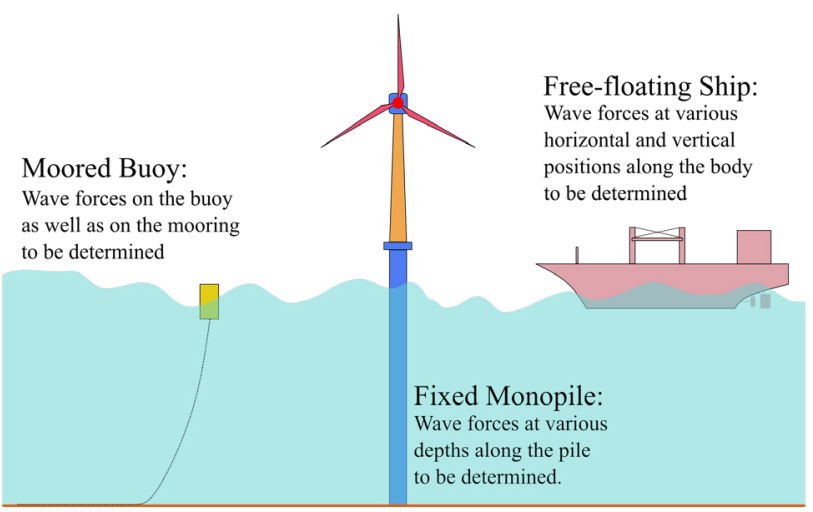

Figure 3. Expected simulation scenarios.

Once the wave parameters such as component frequencies, corresponding amplitudes and phases are determined, they would have to be made available to the body component-model for determination of wave properties at the desired location. Towards this end, an information bus holding the required data is to be specified from which the body component-model may then access this data.

Considering the above general requirements, the system model for integrated simulation may be represented by the block diagram in Figure 4. While the wave, current, and data bus are common components for any ocean engineering simulation, the other components may vary depending upon the scope of the simulation. The rest of this paper is dedicated to the implementation of the component-models for waves and current.

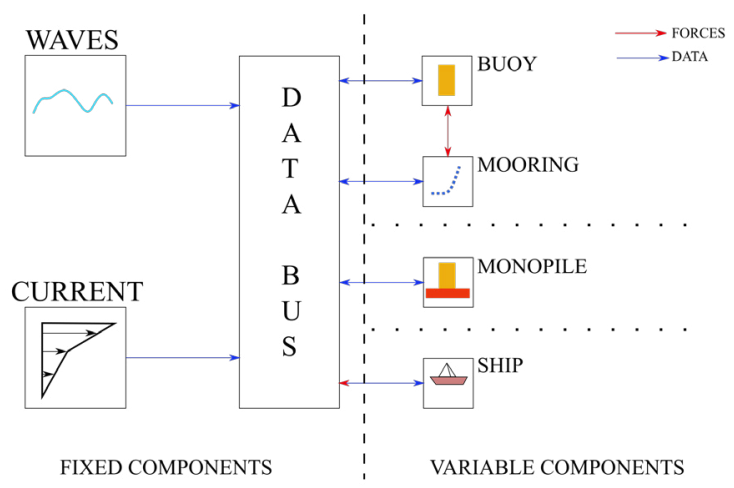

Figure 4. General block diagram for integrated simulation of an ocean engineering system.

Flow-charts provided in the following sub-sections 
have been prepared with ocean engineers, most likely to be unfamiliar with Modelica, in mind, and some elements might appear superfluous to the Modelica savvy reader.

\subsection{Regular-Wave Component-Model}

The height of the regular wave $H_{r}[\mathrm{~m}]$, time period $T_{r}$ [s], water depth $d[\mathrm{~m}]$, water density $\rho_{w}\left[\mathrm{~kg} / \mathrm{m}^{3}\right]$, ramp time $T_{r m p}$ [s], delay time $T_{d e l}$ [s] and the number of frequency components ${ }_{n} \omega_{i}=1$ are specified as parameters in the Regular_Airy_Wave component model. $T_{\text {sim }}[\mathrm{s}]$ is the required duration of simulation.

$T_{r m p}$ is used to ramp the wave height in order to prevent impulse wave loads at the start of the simulation, while $T_{d e l}$ maybe used to start the waves at a specified time into the integrated simulation.

The wave angular frequency $\omega=2 \pi / T$ [rad] and $d$ are passed on as parameters to the function waveNumberIterator, which iterates for the wave number based on the dispersion relation given in (31), and returns the final value to Regular_Airy_Wave.

A data connector WaveDataConnector transmits $d$, $\rho_{w}, \omega, T, k, \varepsilon, \zeta_{0 i}$, and $S S E_{X 0}$ to the data bus which is an expandable connector named the EnvironmentBus. Here, $\varepsilon$ [rad], the phase difference is redundundant for the case of a regular wave and is set to zero, while $S S E_{X 0}$ is the sea surface elevation calculated at $x=0$ using (38).

The algorithm for generation of regular wave parameters is depicted in the flow chart given in Figure 5, and the flow chart for the function waveNumberIterator is given in figure 6. The first value for the wave number iteration is taken to be $k_{0}=\frac{2 \pi}{L_{0}}$, where $L_{0}=\frac{g T^{2}}{2 \pi}[\mathrm{m}]$ is the deep-water wave length as given on p. 66 of (Dean and Dalrymple, 2001).

Equations (37)-(45) can then be used to calculate the wave properties at the required position coordinates, contained in the body component-model, at any required simulation time $t$ [s].

\subsection{Irregular-Wave Component-Model}

The generation of component wave parameters based on the Pierson-Moskowitz spectrum is considered for detailed description. The algorithm for the irregular-wave component-model IRW_PM_RDFCWI is shown in Figure 7.

The water depth $d[\mathrm{~m}]$, significant wave height $H_{s}[\mathrm{~m}]$, the ramp time $T_{r m p}$ [s], the lower cut-off frequency $\omega_{\text {min }}$ $[\mathrm{rad} / \mathrm{s}]$, the upper cut-off frequency $\omega_{\max }[\mathrm{rad} / \mathrm{s}]$, and the number of frequency components to be considered ${ }_{n} \omega_{i}$, are specified as parameter inputs.

The frequency resolution $\Delta \omega=\left(\omega_{\max }-\omega_{\min }\right) /{ }_{n} \omega_{i}$ $[\mathrm{rad} / \mathrm{s}]$ is determined. The component frequency within each frequency interval $\Delta \omega$ is then selected based on a uniform random distribution by the function frequencySelector.

To generate a vector of random numbers, a function randomNumberGenerator based on the Modelica.Math.Random.Generators.Xorshift64star

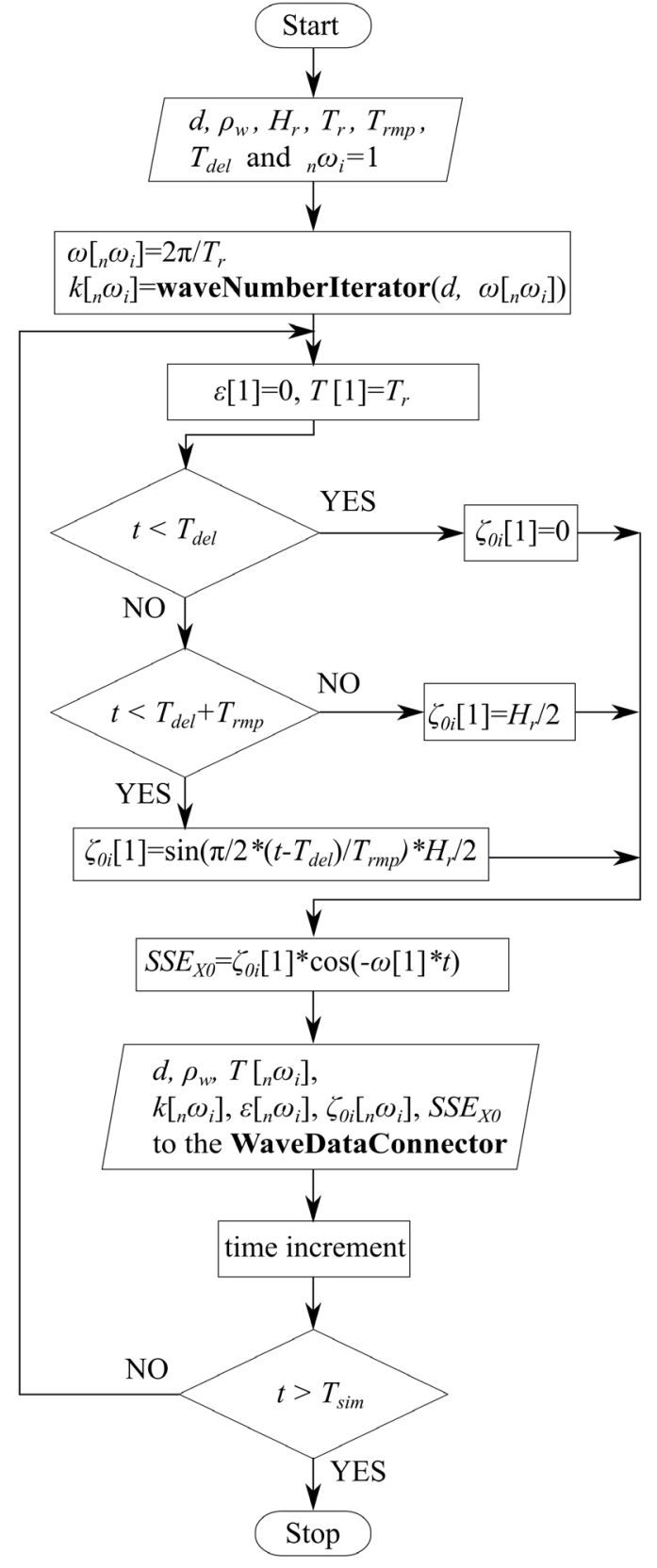

Figure 5. Flow chart for regular-wave component-model.

number generator, with a for loop included, to return a vector of random numbers of specified size, corresponding to the number of frequency components ${ }_{n} \omega_{i}$, is called. The frequencySelector function is a simple function that shifts the component frequencies randomly within the associated frequency interval based on the generated random numbers $r n d \_s h f t\left[{ }_{n} \omega_{i}\right]$.

Once the component frequencies are identified, the corresponding spectral values are determined by calling the function spectrumGenerator_PM which calculates the 


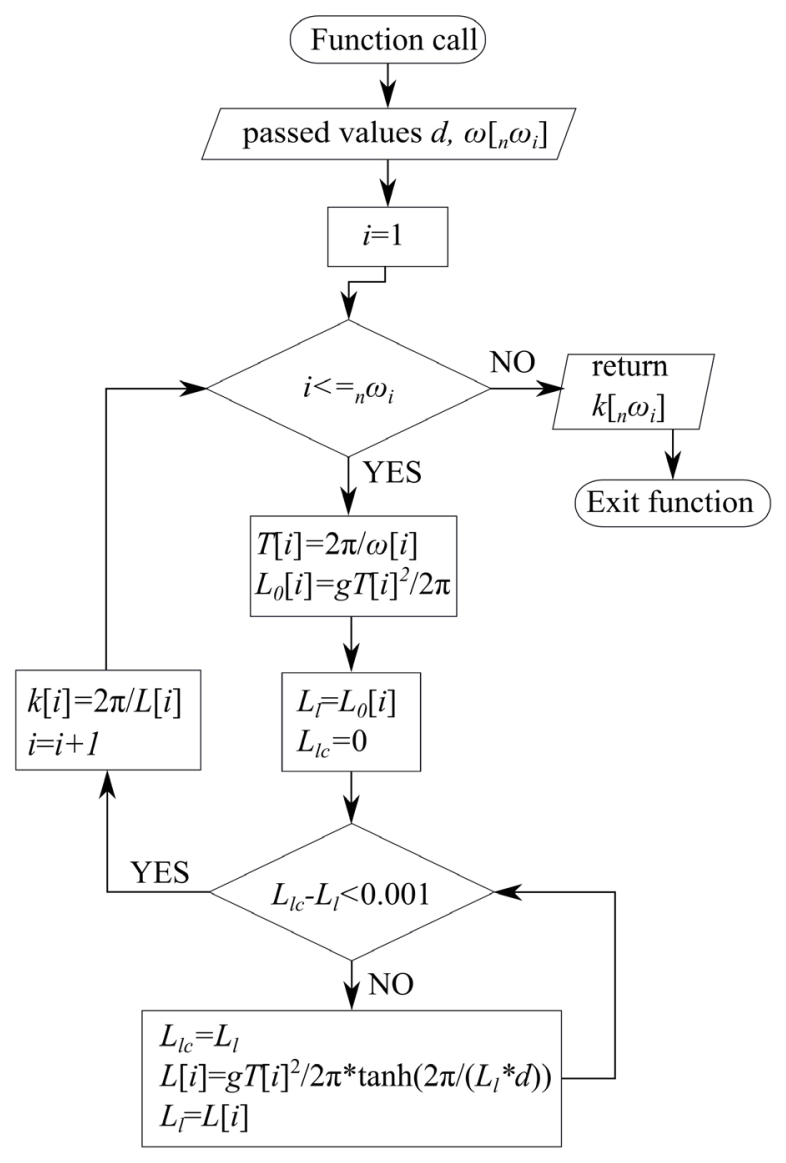

Figure 6. Flow chart for iteration of the wave number.

spectral density values based on the empirical formula

$$
S_{\eta}\left(\omega_{i}\right)=\frac{5 \pi^{4} H_{s}^{2}}{T_{p}^{4} \omega_{i}^{5}} \exp \left(\frac{-20 \pi^{4}}{T_{p}^{4} \omega_{i}}\right) .
$$

Here, $T_{p}[\mathrm{~s}]$ is the peak period of the spectrum, and is related to $H_{s}$ through the relations $T_{p}=\frac{2 \pi}{\omega_{p}}$, and $\omega_{p}^{2}=\frac{0.161 g}{H_{s}}$. $\omega_{p}[\mathrm{rad} / \mathrm{s}]$ is the peak angular frequency; see pp. 105-107 of (Chakrabarti, 1987).

In the future, generation of wave records based on other commonly used sea-spectra may be incorporated by defining the correspoding spectrum generating functions.

The amplitudes of the component waves $\zeta_{0 i}$ are then determined using (47), and corresponding wave numbers $k_{i}$ are determined using the function waveNumberIterator described in Section 3.2. The randomly distributed phases are determined by a second call to the function randomNumberGenerator. This function call returns a vector $\varepsilon\left[{ }_{n} \omega_{i}\right]$ of uniformly distributed random numbers in $(0,1]$ and hence, the associated phase difference is expressed as $2 \pi \varepsilon[\mathrm{rad}]$.

Having determined all the required parameters, the sea surface elevation at $x=0, S S E_{X 0}[\mathrm{~m}]$, is then calculated using the formula given in (48). The values are then linked

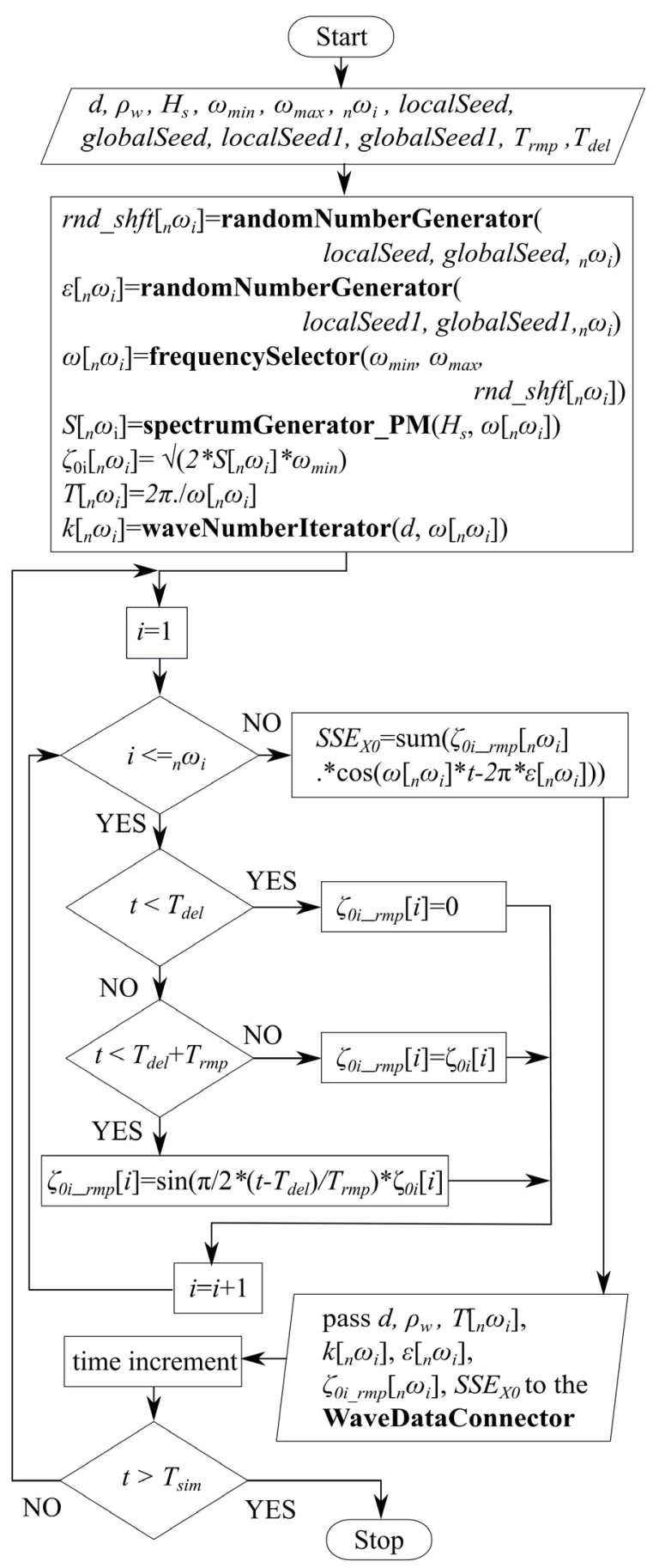

Figure 7. Flow chart for the irregular-wave component-model.

to the expandable connector EnvironmentBus using the WaveDataConnector as described in Section 3.2.

\subsection{Component-Model of Depth-Varying Cur- rent}

The component-model for current is a simple block which produces as its output two vectors $z c g[n]$ and $U c g[n] . z c g$ contains the co-ordinate information and $U c g$ contains the 
corresponding current velocities. The parameters specified are the $z c g[n]$ which is a vector containing the $n$ depth positions where current velocities are defined, $U f[n]$ which is a vector containing the fully developed current values, and the ramp time $T_{\mathrm{rmp}}[\mathrm{s}] . U c g[n]$ holds the instantaneous value of the ramped current. A sinusoidal ramping function is used for smooth ramping. A CurrentDataConnector links the $z c g$ and $U c g$ values to the expandable connector EnvironmentBus. The current velocity at any location may now be computed by the different body component-models by interpolation.

\section{Results}

All results presented below are based on outputs of the above component models. Simulation files are available for download at github.com/ Savin-Viswanathan/Modelica2020-a.

\subsection{Regular Wave}

The simulation model Check_RegularWave under the sample simulations in the above link calculates the wave properties based on the parameters generated by the Regular_Airy_Wave component-model.

Figure 8a shows a sample sea surface elevation at $x=0$ [m] with $T_{d e l}=5$ [s], $T_{r m p}=10[\mathrm{~s}], H_{r}=1[\mathrm{~m}], d=10$ [m] and $T_{r}=3$ [s], for a simulation interval of $0-30$ [s], while Figure $8 \mathrm{~b}$ shows the progressive wave profile for the same wave in the spatial interval 0-30 [m] for different simulation times.

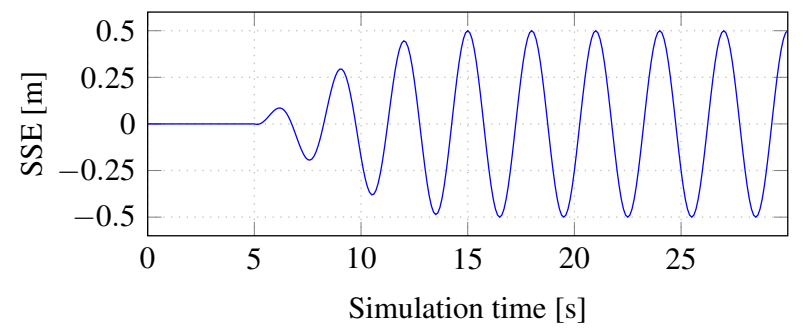

(a) Sea surface elevation at $x=0$ for $t=[0,30] \mathrm{s}$.

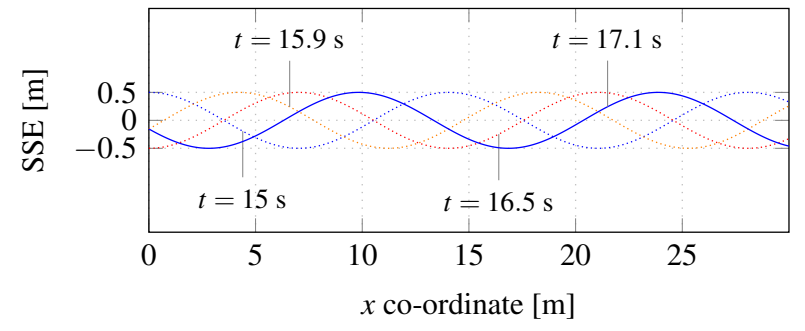

(b) Wave profile at different simulation time steps for $x=[0,30] \mathrm{m}$.

Figure 8. Sea surface elevation and the progressive wave profile.

Figure 9a shows the wave profiles at $t=0$ [s] for different $T_{r}$, and Figure $9 \mathrm{~b}$ shows the trajectory traced by water particles with different mean positions during a complete wave cycle, at different depths, for the different wave periods, in a water depth $d=10[\mathrm{~m}]$. We observe that,
- For $T_{r}=3[\mathrm{~s}], k=0.447414\left[\mathrm{~m}^{-1}\right]$, and $k d>\pi$. The wave is in deep water and the trajectories are circular. The displacements in the vertical and horizontal directions decay exponentially with depth and the particles near the bottom boundary have no horizonatal or vertical displacements.

- For $T_{r}=6[\mathrm{~s}], k=0.129834\left[\mathrm{~m}^{-1}\right]$, and $\frac{\pi}{10}<k d<\pi$. The wave is in intermediate water and the trajectories are elliptical. The displacements in the vertical and horizontal directions decay with depth and the particles near the bottom boundary have only horizontal displacements.

- For $T_{r}=22[\mathrm{~s}], k=0.029246\left[\mathrm{~m}^{-1}\right]$, and $k d<\frac{\pi}{10}$. The wave is in shallow water and the trajectories are elliptical. The displacements in the vertical direction decay linearly with depth, while the horizontal displacement is near constant at all depths.

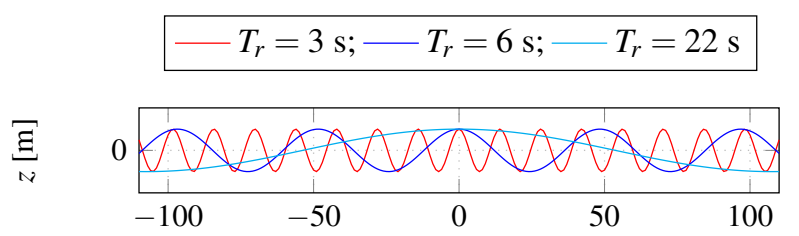

(a) Profiles of waves with different periods.

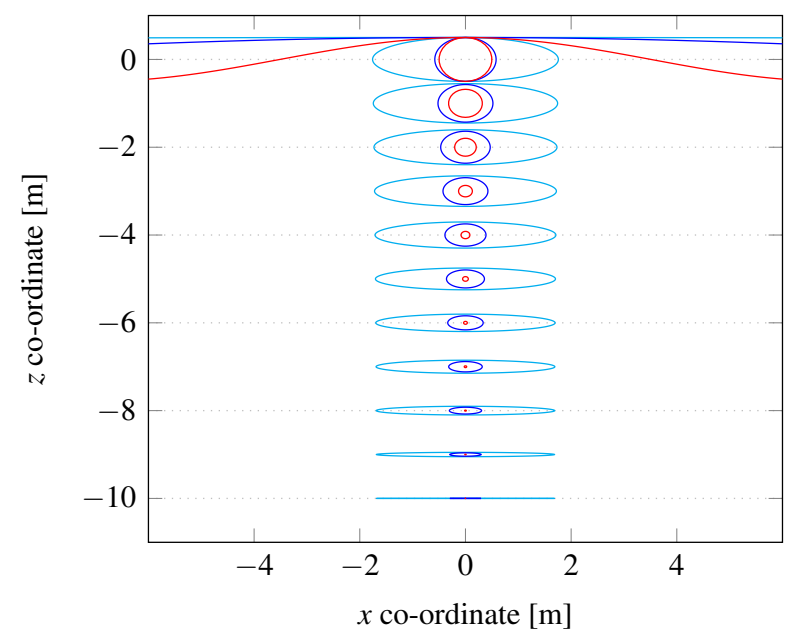

(b) Water particle trajectories of waves with different wave periods.

Figure 9. Wave profiles and water particle trajectories.

Figure 10a shows the intstantaneous wave profile for a regular progressive wave with $T_{r}=6[\mathrm{~s}], H_{r}=1[\mathrm{~m}]$, in a water depth $d=10[\mathrm{~m}]$, when there is a crest at $x=0$ [m]. Figure 10b-10f shows the quiver plots for the instantaneous velocities of water-particles with different mean $z$ co-ordinates, under different $x$ co-ordinates.

An important consideration to keep in mind is that the linearization of the boundary conditions in the derviation of the velocity potential has the effect that the water particle kinematics derived from such a potential does not 


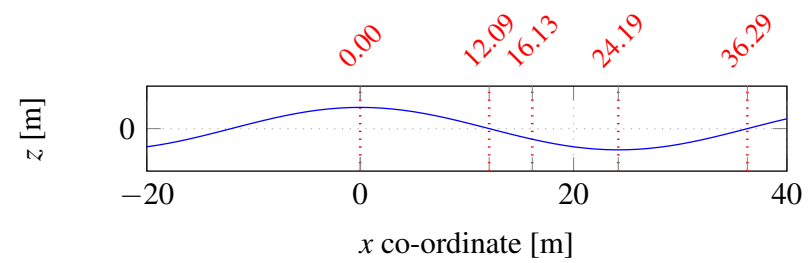

(a) Wave profile and $x$ co-ordinates where the velocities are measured
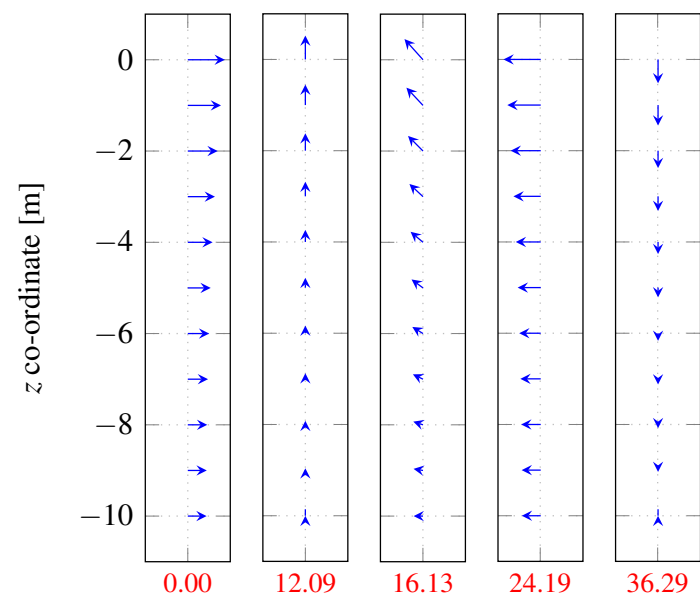

(b)

(c)

(d)

(e)

(f)

Figure 10. Wave profile (a), water-particle velocities (b)-(f).

account for the change of position of the particle within the fluid, and hence the theory cannot give a proper description for flow velocity and acceleration in the region between the still water level and the wave crest and in the void to the wave trough. Extrapolation of the values is, in general, not recommended since the wave forces will be overestimated. A better way is to apply Wheeler stretching or move the profile for velocity and acceleration to the instantaneous sea surface; see p. 221 of (SINTEF, 2014).

Figure 11 shows the pressure distribution at various $x$ co-ordinates for the same wave as above. The dynamic pressure above $z=0[\mathrm{~m}]$ has been calculated using a truncated Taylor series for small positive distances, as given on p. 84 of (Dean and Dalrymple, 2001).

\subsection{Irregular Wave}

Figure 12a depicts a Pierson-Moskowitz spectrum of $H_{s}=$ $1[\mathrm{~m}]$ generated by the spectrumGenerator_PM function, while Figure $12 \mathrm{~b}$ depicts the sea surface elevation for an irregular wave record with 100 frequency components, generated from the spectrum by the IRW_PM_RDFCWI irregular-wave component-model with $T_{r m p}=10[\mathrm{~s}]$, $T_{d e l}=0[\mathrm{~s}], \Delta \omega=\omega_{\min }=0.03141[\mathrm{rad} / \mathrm{s}]$, and $\omega_{\max }=$ $3.141[\mathrm{rad} / \mathrm{s}]$. Figure $12 \mathrm{c}$ shows an expanded view of the same wave record in a shorter time interval, for clarity.

\subsection{Depth-varying Current}

Figure 13 depicts the instantaneous profile for a depth varying current which is based on the output of the Cur-

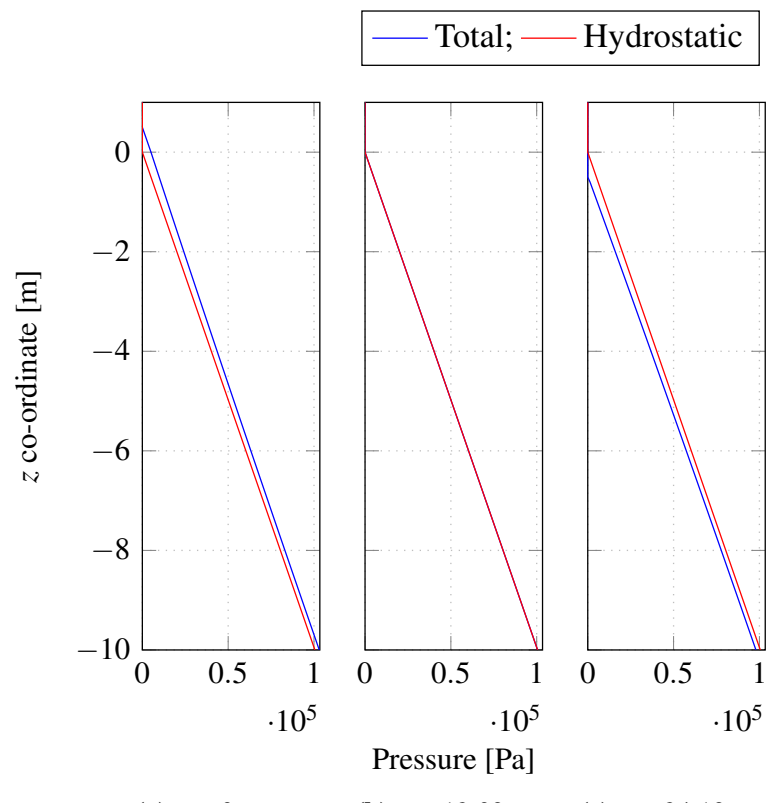

Figure 11. Pressures beneath the wave crest, down-crossing, and trough.

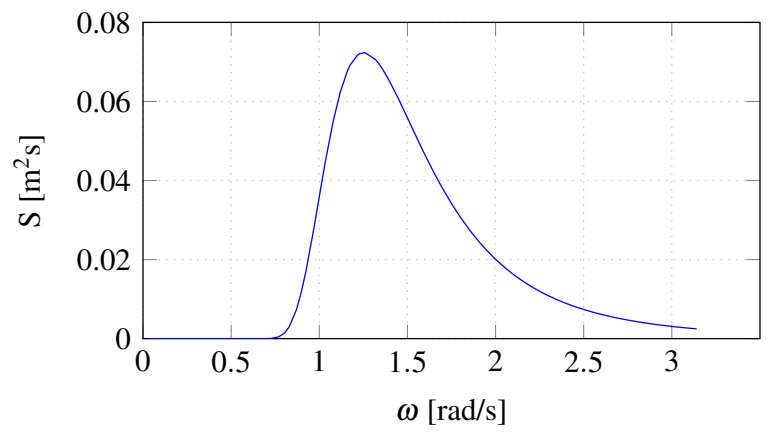

(a) Pierson-Moskowitz spectrum with $H_{s}=1 \mathrm{~m}$.

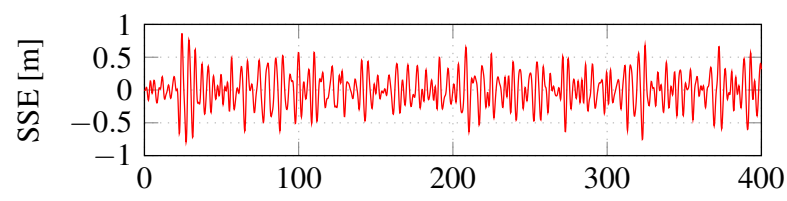

(b) SSE at $x=0 \mathrm{~m}$ for time interval $[0,400] \mathrm{s}$.

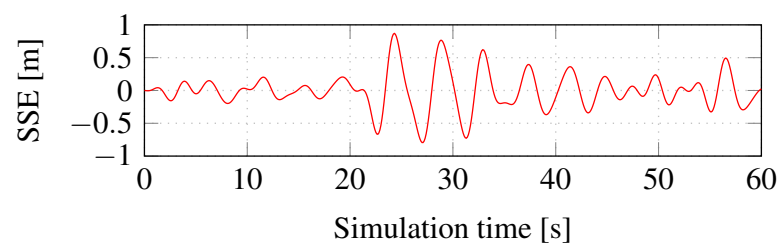

(c) SSE at $x=0 \mathrm{~m}$ for time interval $[0,60] \mathrm{s}$.

Figure 12. Irregular waves.

rentProfile_4pt component-model. The current is ramped up to full value using the parameter $T_{r m p}=5$ [s]. 


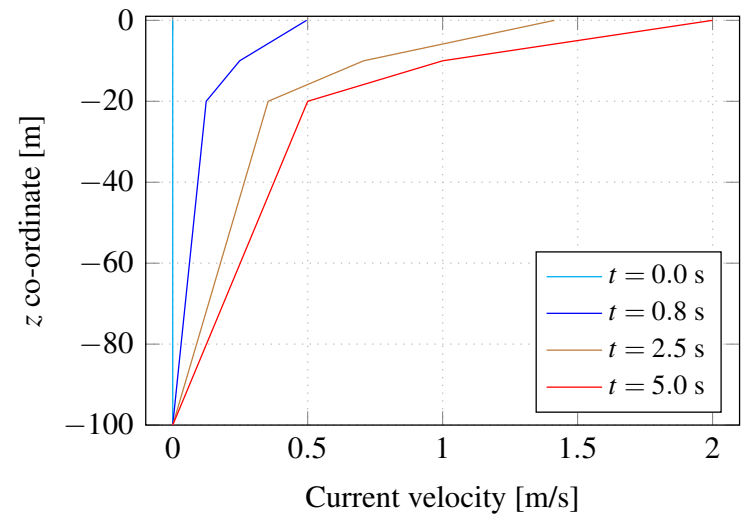

Figure 13. Current profile

\section{Conclusion}

General considerations to be kept in mind while formulating a framework for carrying out integrated simulation of ocean-engineering systems is presented and the algorithms for development of Modelica component-models for the generation of regular and irregular waves are described. The implementation of a simple componentmodel for generation of depth varying current is also presented.

Graphical representation of the wave kinematics and dynamics based on the output of the component-models for regular waves are then presented to show satisfactory agreement with general results discussed in (Dean and Dalrymple, 2001). A sample sea-surface-elevation based on the output of the component-model for irregular waves is presented. Since, within the assumption of linearity, the properties of the irregular wave are a linear combination of the properties of the consitutent regular waves, it is deemed that the output of the irregular wave componentmodel is satisfactory. Graphical representation of the output of the component-model for depth varying current is then presented.

For a better understanding of how these component models perform within an integrated simulation scenario, readers may refer to (Viswanathan and Holden, 2019). The present paper fills in for the lack of theoretical and implementational details for the wave and current component-models in the above work.

Theory and implementation of component-models for non-diffracting floating objects and for mooring forces based on the quasi-static catenary approach, used in (Viswanathan and Holden, 2019), is discussed in (Viswanathan and Holden, 2020), along with comparison of results for the same system modelled in the commonly used ocean-engineering software Orcaflex. Satisfactory agreement of surge/heave responses, and of Morison forces under various combinations of wave and current loading is demonstrated in (Viswanathan and Holden, 2020), and these maybe taken as proof for the correct representation of wave-current kinematics by the component models discussed in this work.

\section{Acknowledgements}

The research in this paper has received funding from the Research Council of Norway, SFI Offshore Mechatronics, project number 90034210 .

\section{References}

Thomas Lykke Andersen and Peter Bak Frigaard. Lecture Notes for the Course in Water Wave Mechanics. Department of Civil Engineering, Aalborg University. DCE Lecture notes, No.24, 2011. URL vbn.aau.dk/en/publications/ lecture-notes-for-the-course-in-waterwave-mechanics (69731932-7a17-47ea-b5576b9e0c81050f). html.

Subratha Kumar Chakrabarti. Hydrodynamics of Offshore Structures. Computational Mechanics Publications, and SpringerVerlag, Dorchester, Great Britain, 1987. ISBN 0-905451-66$\mathrm{X}$.

Robert G. Dean and Robert A. Dalrymple. Water Wave Mechanics for Engineers and Scientists. Allied Publishers Limited, Mumbai, India, 2001. ISBN 81-7764-195-6.

Odd M. Faltinsen. Sea Loads on Ships and Offshore Structures. Cambridge University Press, 1999. ISBN 0-521-45870-6.

Thor I. Fossen. Handbook of Marine Craft Hydrodynamics and Motion Control. John Wiley \& Sons, Chichester, United Kingdom, 2011. ISBN 97-8111-999-1496.

Harald E. Krogstad and Oivind A. Arntsen. Lecture Notes on Linear Wave Theory- Part A-Regular Waves. Norwegian University of Science and Technology, Trondheim, February 2000. URL folk. ntnu. no/oivarn/hercules_ ntnu/LWTcourse/.

SINTEF. Handbook on Design and Operation of Flexible Pipes. 2014. URL sintef.no/en/latest-news/ updated-handbook-on-design-and-operationof-flexible-pipes/.

Alexandra H. Techet. Free Surface Waves- Handout. Massachusetts Institute of Technology, 2005. URL web.mit.edu/2.016/www/handouts/ Free-Surface-Waves.pdf.

Savin Viswanathan and Christian Holden. Towards the development of an ocean engineering library for openmodelica. In Proceedings of the ASME 2019 38th International Conference on Ocean, Offshore and Arctic Engineering., volume 7B: Ocean Engineering, OMAE2019-95054, June, 2019. URL doi .org/10.1115/OMAE2019-95054.

Savin Viswanathan and Christian Holden. Modelica componentmodels for non-diffracting floating objects and quasi-static catenary moorings. Proceedings of the American Modelica Conference, March, 2020. The referring paper and the referred paper are part of the proceedings of the same conference. 